\title{
Continuum damage growth analysis using element free Galerkin method
}

\author{
C O ARUN, B N RAO* and S M SRINIVASAN
}

Structural Engineering Division, Department of Civil Engineering, Indian Institute of Technology Madras, Chennai 600036

e-mail: bnrao@iitm.ac.in

MS received 30 December 2008; revised 14 August 2009; accepted 15 December 2009

\begin{abstract}
This paper presents an elasto-plastic element free Galerkin formulation based on Newton-Raphson algorithm for damage growth analysis. Isotropic ductile damage evolution law is used. A study has been carried out in this paper using the proposed element free Galerkin method to understand the effect of initial damage and its growth on structural response of single and bi-material problems. A simple method is adopted for enforcing EBCs by scaling the function approximation using a scaling matrix, when non-singular weight functions are used over the entire domain of the problem definition. Numerical examples comprising of oneand two-dimensional problems are presented to illustrate the effectiveness of the proposed method in analysis of uniform and non-uniform damage evolution problems. Effect of material discontinuity on damage growth analysis is also presented.
\end{abstract}

Keywords. Damage mechanics; mesh-free method; elasto-plastic element free Galerkin method; essential boundary condition; material discontinuity.

\section{Introduction}

Damage is a progressive physical mechanism which leads to the initiation and growth of micro-voids or micro-cracks. Proper understanding of damage growth is essential to understand the effect of the presence of voids and internal defects on the global response of mechanical/structural system and also on the process which leads these internal defects to final fracture. Evolutionary concept of damage mechanics is proposed by Kachanov (1958), which is based on one-dimensional surface damage variable. Later, an effective stress concept (Rabotnov 1969) associated with strain equivalence principle (Lemaitre 1971) is developed. A well-documented research on damage mechanics is available elsewhere (Lemaitre 1985; Chaboche 1988; Chaboche 1988; Voyiadjis \& Kattan 1990; Lemaitre 1992; Lemaitre \& Desmorat 2005; Voyiadjis \& Kattan 2005). For implementation of damage coupled nonlinear equations in finite element method (FEM), Benallal et al (1988) proposed an integration

*For correspondence 
algorithm based on Newton method, to solve the nonlinear global equilibrium equations as well as the nonlinear local equations obtained by fully implicit integration of the constitutive equations. The applications of different damage models and analysis using numerical tools like FEM are well-explained by Kattan \& Voyiadjis (2002). In recent years mesh-free methods are becoming increasingly popular in the areas of fracture mechanics and related topics.

Mesh-free methods such as smooth particle hydrodynamics (Lucy 1977; Monaghan 1988) diffuse element method (Nayroles et al 1992), element free Galerkin method (EFGM) (Belytschko et al 1994; Lu et al 1994; Belytschko et al 1995), h-p clouds (Duarte \& Oden 1996), partition of unity (Melenk \& Babuska 1996), and reproducing kernel particle method (RKPM) (Liu et al 1995; Liu et al 1997) have been demonstrated particularly attractive for various reasons as they avoid burdensome mesh generation, can easily model the evolution of discontinuities such as cracks and interfaces. Among these methods, EFGM is particularly appealing, due to its simplicity, alleviation of numerical difficulties of mesh entanglement, faster rate of convergence, and formulation that corresponds to well-established FEM. The main drawback that EFGM faced is the problems associated with imposition of the essential boundary conditions (EBCs). Use of non-singular weight functions for moving least square (MLS) approximation will lead to lack of Kronecker delta property of EFGM shape functions, and further leads to difficulties in imposition of EBCs. Researchers came up with different methods to address this problem, like coupled finite element-EFGM (Krongauz \& Belytschko 1996), Lagrange multiplier technique (Dolbow \& Belytschko 1998), penalty method (Liu 2002), full transformation method (Chen \& Wang 2000; Arun et al 2007), extended partition of unity finite element (PUFEM) weight function (Alves \& Rossi 2003), etc. Efficiency of EFGM has been demonstrated in the areas of crack propagation (Rao \& Rahman, 2000; Lu et al 1995), moving boundaries (Li \& Belytschko 2001), large deformation problems (Ponthot \& Belytschko 1998), and others (Nagashima 1999). However, damage analysis using mesh-free techniques has not received much attention. Alves \& Rossi (2003) presented a method which combines the element-free Galerkin with an extended PUFEM, that is able to enforce, in some limiting sense, the essential boundary conditions as done in the FEM and solved some coupled elasto-plastic damage problems using the integration algorithm proposed by Benalall et al (1988). This method requires an iterative scheme for solving global equilibrium equations and also for the local integration of constitutive equations. This will enhance the computational cost required. Moreover, combining extended PUFEM with EFGM can increase the computational complexity of the problem.

In this paper, elasto-plastic element free Galerkin formulation is presented based on Newton-Raphson method for solving the global equilibrium equations and a simple implicit algorithm (Sivakumar \& Voyiadjis 1997) for local integration of constitutive equations. The effect of initial damage on further material behaviour is analysed, since always there exists a probability that an initial damage in the form of micro-cracks or micro-voids be present in all engineering materials and hence materials behave differently from what is expected. The presence of damage in problems with material discontinuities, such as bimaterial problems, is also considered in the present work as different material properties will lead to different rate of damage growth in each of the materials and will affect the interface stresses and strains. In the present work a method using transformation matrix, by which the displacement approximations are scaled along the essential boundaries to get the Kronecker delta properties (Arun et al 2009), is used for EBC and material discontinuity treatment. Isotropic damage evolution law (Lemaitre 1985; Lemaitre \& Desmorat 2005) is used in this study. The paper is organized as follows. Section 2 presents a brief outline of continuum damage theory. Section 3 describes elasto-plastic EFGM formulation for damage mechanics, imposition of 


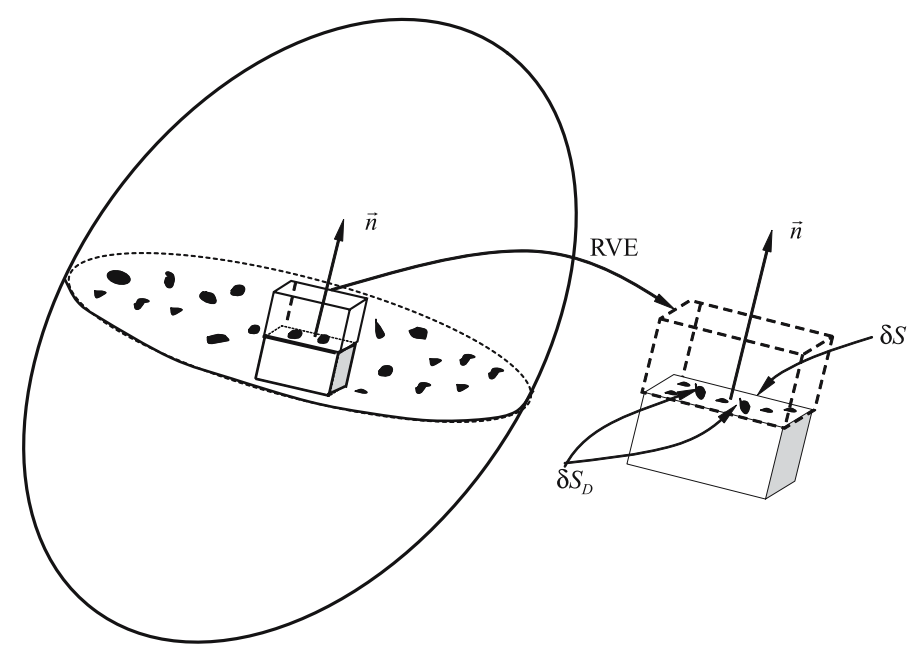

Figure 1. Mechanical representation of damage.

EBCs and state determination procedure. Section 4 outlines the treatment of material discontinuity using the proposed method. Section 5 presents numerical examples to illustrate the performance of the proposed methodology.

\section{Continuum damage theory}

Consider a damaged body as shown in figure 1, in which a representative volume element (RVE) is isolated. Damage variable is physically defined by the surface density of microcracks and intersections of micro-voids lying on a plane cutting RVE of cross section $\delta S$ (Lemaitre 1992; Lemaitre \& Desmorat 2005). Damage variable $D_{(\vec{n})}$, for the plane defined by normal $\vec{n}$ is

$$
D_{(\vec{n})}=\frac{\delta S_{D}}{\delta S}, 0 \leq D(\vec{n}) \leq 1,
$$

where $\delta S_{D}$ is the effective area of the intersections of all micro-cavities or micro-cracks that lie in $\delta S$. An isotropic damage variable (Kachanov 1958; Lemaitre 1985) which is independent of normal, assumes damage is equally distributed in all directions, is defined as

$$
D=\frac{\delta S_{D}}{\delta S}
$$

where $D$ is scalar. The effective stress tensor (Rabotnov 1969; Lemaitre 1985), based on stress acting on resisting area, for a multi-axial case with isotropic damage is defined as

$$
\tilde{\sigma}_{i j}=\frac{\sigma_{i j}}{1-D},
$$

where $\tilde{\boldsymbol{\sigma}}$ is the effective stress tensor and $\boldsymbol{\sigma}$ is the stress tensor.

The principle of strain equivalence (Lemaitre 1971; Lemaitre 1985), which assumes that the strain behaviour is modified by damage only through the effective stress, states that the strain 
constitutive equations for a damaged material can be derived using the same formulations used for an undamaged material except that the stress is replaced by the effective stress. The thermodynamics of damage along with elasticity and plasticity is included in the isotropic unified damage law (Lemaitre 1992; Lemaitre \& Desmorat 2005), which is briefly discussed below.

According to isotropic unified damage law, the main variable governing the damage evolution is the damage rate and the associated variable is the energy density release rate (Lemaitre \& Desmorat 2005). The dissipative potential function, $F$ is defined as

$$
F=f+F_{\chi}+F_{D}
$$

where $f$ is the plasticity criterion function defined as,

$$
f=(\tilde{\boldsymbol{\sigma}}-\chi)_{e q}-\kappa-\sigma_{Y},
$$

where $\kappa$ is the isotropic hardening stress variable, $\chi$ is the back stress related to kinematic hardening, $\sigma_{Y}$ is the yield stress and

$$
(\tilde{\boldsymbol{\sigma}}-\chi)_{e q}=\sqrt{\frac{3}{2}\left(\tilde{\sigma}_{i j}^{D}-\chi_{i j}^{D}\right)\left(\tilde{\sigma}_{i j}^{D}-\chi_{i j}^{D}\right)} .
$$

$F_{\chi}$ is the nonlinear kinematic hardening term modelled by

$$
F_{\chi}=\frac{3 \gamma}{4 c} \chi_{i j} \chi_{i j}
$$

with $c$ and $\gamma$ are the temperature dependent material parameters. For linear kineamatic hardening $\gamma$ is set to zero. $F_{D}$ is the damage potential defined as

$$
F_{D}=\frac{S}{(s+1)(1-D)}\left(\frac{Y}{S}\right)^{s+1},
$$

where $S$ and $s$ are the material parameters which depends on temperature, and $Y$ is the energy density rate given by

$$
Y=\frac{\tilde{\sigma}_{e q}^{2} R_{v}}{2 E_{0}},
$$

where $R_{v}$ is the triaxiality function defined by

$$
R_{v}=\frac{2}{3}(1+v)+3(1-2 v)\left(\frac{\sigma_{H}}{\sigma_{e q}}\right)^{2}
$$

where $v$ is the poison ratio, $\sigma_{H}=\sigma_{k k} / 3$ is the hydrostatic stress, $\sigma_{e q}=\sqrt{\frac{3}{2} \sigma_{i j}^{D} \sigma_{i j}^{D}}$ is the von Mises equivalent stress with the stress deviator, $\sigma_{i j}^{D}=\sigma_{i j}-\sigma_{H} \delta_{i j}$, and $\tilde{\sigma}_{e q}=\sigma_{e q}(1-D)$ is the effective von Mises stress. $E_{0}$ is the Young's modulus of virgin material. 
The evolution laws of internal variables are derived as

$$
\begin{aligned}
\dot{\varepsilon}_{i j}^{p} & =\dot{\lambda} \frac{\partial F}{\partial \tilde{\sigma}_{i j}}=\dot{\lambda} \frac{\partial f}{\partial \tilde{\sigma}_{i j}}, \\
\dot{\alpha}_{i j} & =-\dot{\lambda} \frac{\partial F}{\partial \chi_{i j}}, \\
\dot{v} & =-\dot{\lambda} \frac{\partial F}{\partial \kappa}
\end{aligned}
$$

and

$$
\dot{D}=\dot{\lambda} \frac{\partial F}{\partial Y}=\dot{\lambda} \frac{\partial F_{D}}{\partial Y},
$$

where $\dot{\lambda}$ is the plastic multiplier obtained by the consistency conditions, $f=0$ and $\dot{f}=0$. $\boldsymbol{\varepsilon}^{p}$ is plastic strain tensor, $\boldsymbol{\alpha}$ is kinematic hardening state variable, and $v$ is isotropic hardening state variable.

Using equations (8) and (14) the damage constitutive equation is derived as

$$
\begin{aligned}
& \dot{D}= \begin{cases}\left(\frac{Y}{S}\right)^{s} \frac{\dot{\lambda}}{(1-D)}, & \text { if } p>p_{D} \\
0, & \text { if not }\end{cases} \\
& D=D_{c} \rightarrow \text { meso-crack initiation, }
\end{aligned}
$$

where $\dot{D}$ is the damage rate, $p$ is the accumulated plastic strain defined by $p=\sqrt{\frac{2}{3} \varepsilon_{i j}^{p} \varepsilon_{i j}^{p}}$ for von Mises yield criteria, and $D_{c}$ is the critical damage value at which the meso-crack initiations occur, which is a material parameter. The damage threshold, $p_{D}$ which is the accumulated plastic strain at which damage starts, is defined as

$$
\begin{aligned}
& p_{D}=\varepsilon_{p D} \quad \text { for monotonic loading } \\
& p_{D}=\varepsilon_{p D}\left(\frac{\sigma_{u}-\sigma_{f}^{\infty}}{\frac{\sigma_{e q \max }+\sigma_{e q \min }}{2}-\sigma_{f}^{\infty}}\right)^{m} \text { for cyclic loading, }
\end{aligned}
$$

where $\varepsilon_{p D}$ is the damage threshold in pure tension, $m$ is the correction parameter, $\sigma_{u}$ is the ultimate stress and $\sigma_{f}^{\infty}$ is the asymptotic fatigue stress.

In the present study an initial damage is assumed to present in the material and so a realistic assumption that damage will start to grow once the hardening starts is considered. However damage closure effect is not considered in this study.

\section{Elasto-plastic EFGM formulation for damage mechanics}

\subsection{Variational formulation and discretization}

For small displacements in two-dimensional problems with isotropic damage, the equilibrium equations and boundary conditions can be written in terms of effective stress as

$$
\begin{aligned}
& \boldsymbol{\nabla} \cdot((\mathbf{1}-D) \tilde{\boldsymbol{\sigma}})+\boldsymbol{b}=0 \text { in } \Omega, \\
& ((\mathbf{1}-D) \tilde{\boldsymbol{\sigma}}) \cdot \boldsymbol{n}=\overline{\boldsymbol{t}} \text { on } \Gamma_{t} \text { (natural boundary conditions), } \\
& \boldsymbol{u}=\overline{\boldsymbol{u}} \text { on } \Gamma_{u} \text { (essential boundary conditions), }
\end{aligned}
$$


where, $\tilde{\boldsymbol{\sigma}}$ is the effective stress vector, $\boldsymbol{b}$ is the body force vector, $\overline{\boldsymbol{t}}$ and $\overline{\boldsymbol{u}}$ are the vectors of prescribed surface tractions and displacements, respectively, $\boldsymbol{n}$ is a unit normal to domain $\Omega, \Gamma_{t}$ and $\Gamma_{u}$ are the portions of boundary, $\Gamma$ where tractions and displacements are prescribed, respectively, and $\boldsymbol{\nabla}^{T}=\left\{\partial / \partial x_{1}, \partial / \partial x_{2}\right\}$ is the vector of gradient operators. The variational form of the equation (17) can be written as

$$
\int_{\Omega}(1-D) \delta \boldsymbol{\varepsilon}^{T} \tilde{\boldsymbol{\sigma}} d \Omega-\int_{\Omega} \delta \boldsymbol{u}^{T} \boldsymbol{b} d \Omega-\int_{\Gamma_{f}} \delta \boldsymbol{u}^{T} \overline{\boldsymbol{t}} d \Gamma=0,
$$

where $\delta \boldsymbol{u}$ and $\delta \boldsymbol{\varepsilon}$ are virtual displacement increment and strain increment respectively. Using meshless discretization of the domain, the MLS (Lancaster \& Salkauskas 1981) approximation of displacement vector $\boldsymbol{u}$, is given by (Belytschko et al 1994; Rao \& Rahman 2000)

$$
u^{h}(x)=\sum_{I=1}^{n} \Phi_{I}(\boldsymbol{x}) d_{I}=\boldsymbol{\Phi}^{T}(\boldsymbol{x}) \boldsymbol{d},
$$

where $n$ is the number of nodes influencing $\boldsymbol{x}, \boldsymbol{\Phi}^{T}(\boldsymbol{x})=\left\{\Phi_{1}(\boldsymbol{x}), \Phi_{2}(\boldsymbol{x}), \ldots, \Phi_{n}(\boldsymbol{x})\right\}$, with $\Phi_{I}(\boldsymbol{x})$ representing the shape function of MLS approximation corresponding to node $I$, and $\boldsymbol{d}^{T}=\left\{d_{1}, d_{2}, \ldots, d_{n}\right\}$ with $d_{I}$ representing the nodal parameter (not the nodal values of $u^{h}(\boldsymbol{x})$ ) for node $I$. Substituting of equation (21) into equation (20) results in

$$
\int_{\Omega}(1-D) \boldsymbol{B}^{T} \tilde{\boldsymbol{\sigma}} d \Omega=\boldsymbol{R}
$$

where $\boldsymbol{B}$ is the strain displacement matrix. For node $I, \boldsymbol{B}$ is given by

$$
\boldsymbol{B}_{I}=\left[\begin{array}{cc}
\Phi_{I, 1} & 0 \\
0 & \Phi_{I, 2} \\
\Phi_{I, 2} & \Phi_{I, 1}
\end{array}\right]
$$

and $\boldsymbol{R}$ is the external equivalent force vector, defined by

$$
\boldsymbol{R}_{I}=\int_{\Omega} \Phi_{I} \boldsymbol{b} d \Omega-\int_{\Gamma_{f}} \Phi_{I} \bar{t} d \Gamma \in \Re^{2} .
$$

For analysis which includes material nonlinearity, equation (22) becomes a nonlinear function of the displacement and so a nonlinear function of the nodal parameter vector, $\boldsymbol{d}$. Hence iterative methods are needed for solving the equation (22). Since an elasto-plastic constitutive relation depends on deformation history, an incremental analysis following an actual variation of external forces should be used to trace the variation of displacement, strain, and stress along with external forces. Newton-Raphson method (Chen \& Han 1988), is used to solve the global equilibrium equations where as a simple implicit scheme (Sivakumar \& Voyiadjis 1997 ) is used to solve the constrained elasto-plastic damage evolution equations and for updating variables.

The load $\boldsymbol{R}$, is applied incrementally to the mechanical/structural system. Let, load at the $(r+1)$ th step is ${ }^{r+1} \boldsymbol{R}$, which can be expressed as

$$
{ }^{r+1} \boldsymbol{R}={ }^{r} \boldsymbol{R}+{ }^{r+1} \Delta \boldsymbol{R} .
$$

Corresponding to the load increment, equation (22) can be written as

$$
{ }^{r+1} \boldsymbol{F}={ }^{r+1} \boldsymbol{R},
$$


where ${ }^{r+1} \boldsymbol{F}$ is the internal force vector, which is a function of the nodal displacement parameter ${ }^{r+1} \boldsymbol{d}$ and can be written as

$$
{ }^{r+1} \boldsymbol{F}\left({ }^{r+1} \boldsymbol{d}\right)=\int_{\Omega}\left(1-{ }^{r+1} D\left({ }^{r+1} \boldsymbol{d}\right)\right) \boldsymbol{B}^{T r+1} \tilde{\boldsymbol{\sigma}}\left({ }^{r+1} \boldsymbol{d}\right) d \Omega,
$$

where

$$
{ }^{r+1} \boldsymbol{d}={ }^{r} \boldsymbol{d}+\Delta \boldsymbol{d} .
$$

Equation (26) can be rewritten as

$$
\Psi\left({ }^{r+1} \boldsymbol{d}\right)={ }^{r+1} \boldsymbol{F}\left({ }^{r+1} \boldsymbol{d}\right)-{ }^{r+1} \boldsymbol{R} .
$$

First order Taylor series expansion of $\Psi$ at ${ }^{r+1} \boldsymbol{d}^{i}$, where ${ }^{r+1} \boldsymbol{d}^{i}$ is the $i$ th approximation of ${ }^{r+1} \boldsymbol{d}$, results in

$$
\left.\frac{\partial \boldsymbol{F}}{\partial \boldsymbol{d}}\right|_{r+1} \Delta \boldsymbol{d}^{i+1}+{ }^{r+1} \boldsymbol{F}^{i}-{ }^{r+1} \boldsymbol{R}=0 .
$$

Using equation (27)

$$
\begin{aligned}
\left.\frac{\partial \boldsymbol{F}}{\partial \boldsymbol{d}}\right|_{r+1} & =\left.\int_{\Omega} \boldsymbol{B}^{T}\left((1-D) \frac{\partial \tilde{\boldsymbol{\sigma}}}{\partial \boldsymbol{d}}-\tilde{\boldsymbol{\sigma}} \frac{\partial D}{\partial \boldsymbol{d}}\right)\right|_{r+1_{d^{i}}} d \Omega \\
& =\left.\int_{\Omega} \boldsymbol{B}^{T}\left((1-D) \boldsymbol{C}^{e p}\right)\right|_{r+1_{d^{i}}} \boldsymbol{B} d \Omega-\left.\int_{\Omega} \boldsymbol{B}^{T}\left(\tilde{\boldsymbol{\sigma}} \frac{\partial D}{\partial \boldsymbol{\varepsilon}}\right)\right|_{r+1_{d^{\prime}}} \boldsymbol{B} d \Omega,
\end{aligned}
$$

where $\boldsymbol{C}^{e p}$ is elasto-plastic matrix. Using equation (31), the equation (30) can be rewritten in the form

$$
{ }^{r+1} \boldsymbol{K}^{i} \Delta \boldsymbol{d}^{i+1}={ }^{r+1} \boldsymbol{R}-{ }^{r+1} \boldsymbol{F}^{i},
$$

where

$$
{ }^{r+1} \boldsymbol{K}^{i}=\left.\int_{\Omega} \boldsymbol{B}^{T}\left((1-D) \boldsymbol{C}^{e p}\right)\right|_{r+1_{d^{i}}} \boldsymbol{B} d \Omega-\left.\int_{\Omega} \boldsymbol{B}^{T}\left(\tilde{\boldsymbol{\sigma}} \frac{\partial D}{\partial \boldsymbol{\varepsilon}}\right)\right|_{r+1_{d^{i}}} \boldsymbol{B} d \Omega .
$$

The second term in equation (33) may lead to numerical instabilities, when it dominates over the first term, while solving equation (32). But Newton-Raphson method gives the freedom to modify the stiffness matrix by neglecting the second term at the cost of number of iterations. In the present work equation (33) is modified as

$$
{ }^{r+1} \boldsymbol{K}^{i}=\left.\int_{\Omega} \boldsymbol{B}^{T}\left((1-D) \boldsymbol{C}^{e p}\right)\right|_{r+1_{d i}} \boldsymbol{B} d \Omega
$$

\subsection{Imposition of essential boundary conditions}

EFGM shape function $\Phi_{I}(\boldsymbol{x})$ does not satisfy the Kronecker delta property i.e. $\Phi_{I}\left(\boldsymbol{x}_{J}\right) \neq \delta_{I J}$. Therefore $u_{i}^{h}\left(\boldsymbol{x}_{J}\right) \neq d_{J}^{i}$ and $\Delta u_{i}^{h}\left(\boldsymbol{x}_{J}\right) \neq \Delta d_{J}^{i}$, which complicates the imposition of EBCs. For enforcement of the essential boundary conditions, in this study a simple method is adopted. 


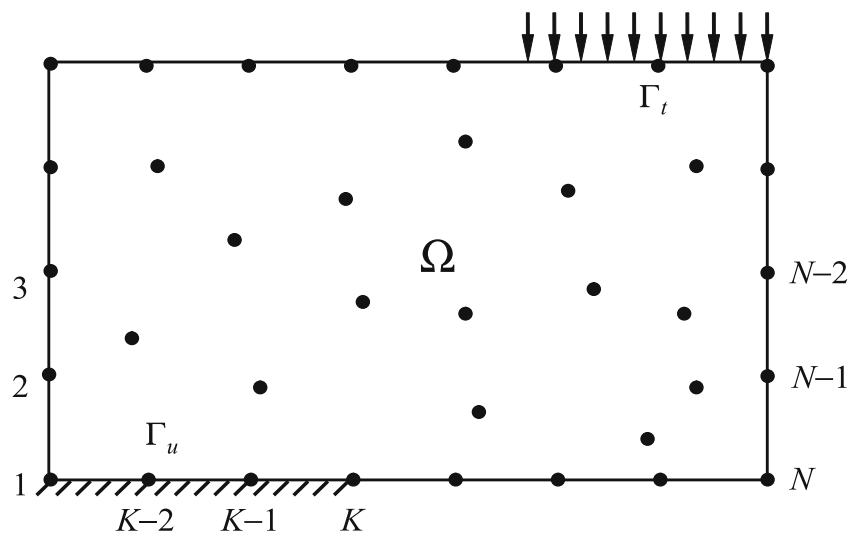

Figure 2. Meshless discretization of domain $\Omega$.

In this method, unlike the general EFGM formulation, MLS approximation is scaled along the essential boundaries by a scaling matrix. For the domain discretized using meshless nodes as shown in figure 2 , the condition $u_{i}^{h}\left(\boldsymbol{x}_{J}\right)=d_{J}^{i}$ is satisfied along the essential boundary $\Gamma_{u}$ through a scaled transformation which modifies equation (21) as

$$
u_{i}^{h}\left(\boldsymbol{x}_{J}\right)=\sum_{I_{1}=1}^{N} \sum_{I_{2}=1}^{N} \Phi_{I_{1}}\left(\boldsymbol{x}_{J}\right) \Lambda_{I_{1} I_{2}}^{\prime-1} d_{I_{2}}^{i}=\Phi_{J}^{i T} \Lambda^{\prime-1} \boldsymbol{d},
$$

and so

$$
\Delta u_{i}^{h}\left(\boldsymbol{x}_{J}\right)=\boldsymbol{\Phi}_{J}^{i T} \boldsymbol{\Lambda}^{-1} \boldsymbol{d},
$$

where $N$ is the total number of nodal points in the domain $\Omega, \boldsymbol{d}$ is the nodal parameters not the nodal values and

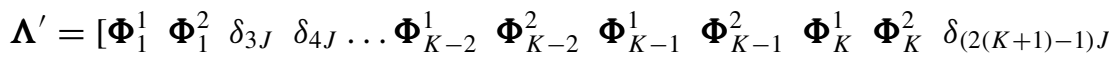

$$
\begin{aligned}
& \left.\delta_{(2(K+1)) J} \ldots \delta_{(2 N-1) J} \delta_{(2 N) J}\right]^{T} \in \mathcal{L}\left(\Re^{2 N} \times \Re^{2 N}\right),
\end{aligned}
$$

is the scaled transformation matrix which includes shape function values associated with the nodes along the essential boundary, where $\delta_{I J}=1$, when $I=J$, and $\delta_{I J}=0$ when $I \neq J$. Using equations (35) and (36), equation (32) can be modified as

$$
{ }^{r+1} \overline{\boldsymbol{K}}^{i} \Delta \boldsymbol{d}^{i+1}=\overline{\boldsymbol{F}}
$$

with

$$
{ }^{r+1} \overline{\boldsymbol{K}}^{i}=\boldsymbol{\Lambda}^{-T r+1} \boldsymbol{K}^{i} \boldsymbol{\Lambda}^{\prime-1},
$$

and

$$
\overline{\boldsymbol{F}}=\boldsymbol{\Lambda}^{\prime-T}\left({ }^{r+1} \boldsymbol{R}-{ }^{r+1} \boldsymbol{F}^{i}\right) .
$$

The discretized equation system in equation (38) can be solved for the $(i+1)$ th increment in nodal parameter after applying the appropriate essential boundary conditions given in equation (19) by adopting the procedures similar to that in finite element analysis. The corresponding increment in nodal values can be obtained from equation (36). Once $\Delta \boldsymbol{d}^{i+1}$ is evaluated, 
the corresponding strain, stress and damage increment can be found out using the procedure described further. During Newton-Raphson iteration, an inaccurate transition to plastic state may occur especially if the load step is too large. This should result in large imbalance, and the following iterations should in general be able to correct it. However, any plastic strain that was accumulated during the iteration will still be there and in later iterations will give an erroneous yield stress (Bhatti 2006). To avoid this problem, instead of using the displacement increment from the current iteration, the total displacement increment from the beginning of the current load step should be used. Thus

$$
\Delta \boldsymbol{d}^{i+1}=\sum_{i=1}^{i+1} \Delta \boldsymbol{d}^{j} .
$$

Then the total strain increment,

$$
\Delta \varepsilon^{i+1}=B^{T} \Lambda^{\prime-1} \Delta d^{i+1} .
$$

Once the strain increment is calculated, the other state variables like stress, plastic strain and damage at the end of new load step is to be found. The state determination procedure is briefly outlined below.

\subsection{Procedure for state determination}

For a given Gauss point, with known increment in strain $(\Delta \varepsilon)$, and the previous state $\left(\tilde{\boldsymbol{\sigma}}, \boldsymbol{\varepsilon}, \boldsymbol{\chi}, \kappa\right.$, and $\left.\boldsymbol{\varepsilon}^{p}\right)$ the basic steps in computing a new state are as follows:

1. Compute the elastic predictor, and trial stress increment using,

$$
\begin{gathered}
\Delta \tilde{\boldsymbol{\sigma}}^{e}=\boldsymbol{C} \Delta \boldsymbol{\varepsilon} \\
\tilde{\boldsymbol{s}}=\tilde{\boldsymbol{\sigma}}+\Delta \tilde{\boldsymbol{\sigma}}^{e} .
\end{gathered}
$$

2. Evaluate the yield function value $f(\tilde{\boldsymbol{s}}, \boldsymbol{\chi}, \kappa)$. For a previously elastic Gauss point continue with step 3, otherwise go to step 4 .

3. For a previously elastic Gauss point, there are two possibilities,

(a) Gauss point remains to be elastic i.e. $f(\tilde{\boldsymbol{s}}, \boldsymbol{\chi}, \kappa) \leq 0$ and ${ }^{r+1} \tilde{\boldsymbol{\sigma}}^{i+1}=\tilde{\boldsymbol{s}}$ and other state variables, related to plastic part remains same as in the previous load step. Go to step 7 .

(b) $f(\tilde{\boldsymbol{s}}, \boldsymbol{\chi}, \kappa)>0$ with a transition from elastic to plastic state. Locate stress at yield point by solving $f\left(\tilde{\boldsymbol{\sigma}}+\boldsymbol{\rho} \boldsymbol{\Delta} \boldsymbol{\sigma}^{e}, \boldsymbol{\chi}, \kappa\right)=0$ where $\rho$ is the fraction of strain increment that takes the stress to yield stress level. $\rho \Delta \varepsilon$ is the strain increment that takes stress to yield level and $(1-\rho) \Delta \boldsymbol{\varepsilon}$ is called as elasto-plastic strain increment. Continue to step 5.

4. For a previous yielded Gauss point there are three possibilities;

(a) It continues to yield plastically. Then $\rho=0$ and continue to step 5 .

(b) It unloads elastically, with $f(\tilde{\boldsymbol{s}}, \boldsymbol{\chi}, \kappa) \leq 0$. Then ${ }^{r+1} \tilde{\boldsymbol{\sigma}}^{i+1}=\tilde{\boldsymbol{s}}$ and go to step 7 .

(c) It unloads by moving inside the yield surface and eventually ends up with a stress out side the yield surface, which requires to find $\rho$ and then continue to step 5 . 
5. In this step the stresses corresponding to $(1-\rho) \Delta \varepsilon$ are to be found out. The available literature on damage mechanics uses the integration scheme proposed by Benallal et al 1988, which requires an iteration for yield surface drift correction, to minimize the errors that arise due the replacement of differential quantities to finite difference quantities. In the present work a simple implicit scheme (Sivakumar \& Voyiadjis 1997), which uses single point integration without any iteration, is used. The final state for which the yield condition to be satisfied is used to obtain the discrete consistency condition,

$$
f\left(\tilde{\boldsymbol{\sigma}}+\rho \Delta \boldsymbol{\sigma}^{e}+\Delta \tilde{\boldsymbol{\sigma}}, \boldsymbol{\chi}+\Delta \boldsymbol{\chi}, \kappa+\Delta \kappa\right)=0 .
$$

For an associated flow rule, the evolution equations given in equations (11)-(14) can be rewritten in incremental form as

$$
\begin{aligned}
& \Delta \boldsymbol{\varepsilon}^{p}=\Delta \lambda \frac{\partial f}{\partial \tilde{\boldsymbol{\sigma}}} \\
& \Delta \boldsymbol{\chi}=-h \Delta \lambda \frac{\partial F}{\partial \chi} \\
& \Delta \kappa=-H \Delta \lambda \frac{\partial F}{\partial \kappa} \\
& \Delta D=\Delta \lambda \frac{\partial F_{D}}{\partial Y}
\end{aligned}
$$

where $h$ and $H$ are kinematic and isotropic hardening modulus respectively. The elastoplastic constitutive equation can be written as

$$
\Delta \tilde{\boldsymbol{\sigma}}=\boldsymbol{C}\left(\Delta \bar{\varepsilon}-\Delta \varepsilon^{p}\right),
$$

where $\Delta \bar{\varepsilon}=(1-\rho) \Delta \varepsilon$. Substituting equations (45)-(49) into equation (44), will lead to a polynomial in $\Delta \lambda$. For the von Mises yield criteria, which is used in the present study, the polynomial will be a quadratic equation in $\Delta \lambda$ with coefficients in terms of initial quantities (Sivakumar \& Voyiadjis 1997). To solve the equation (44) for $\Delta \lambda$ and using equations (45)-(49) update all the variables as

$$
\begin{aligned}
& { }^{r+1} \tilde{\boldsymbol{\sigma}}^{i+1}=\tilde{\boldsymbol{\sigma}}+\rho \Delta \boldsymbol{\sigma}^{e}+\Delta \tilde{\boldsymbol{\sigma}} \\
& { }^{r+1} \boldsymbol{\varepsilon}^{i+1}=\boldsymbol{\varepsilon}+\Delta \boldsymbol{\varepsilon} \\
& { }^{r+1} \boldsymbol{\varepsilon}^{p i+1}=\boldsymbol{\varepsilon}^{p}+\Delta \boldsymbol{\varepsilon}^{p} \\
& { }^{r+1} \chi^{i+1}=\chi+\Delta \boldsymbol{\chi} \\
& { }^{r+1} \kappa^{i+1}=\kappa+\Delta \kappa \\
& { }^{r+1} D^{i+1}=D+\Delta D .
\end{aligned}
$$

6. For an associated flow rule, the elasto-plastic stiffness matrix $\boldsymbol{C}^{e p}$ is evaluated at each iteration using the method described by Chen \& Han (1988).

7. Check for convergence with new state values. Force equilibrium and internal energy criteria can be used (Chen \& Han 1988; Bhatti 2006). If the prescribed tolerance is achieved, Newton-Raphson iterations can be stopped and can be proceeded to the next load step. 

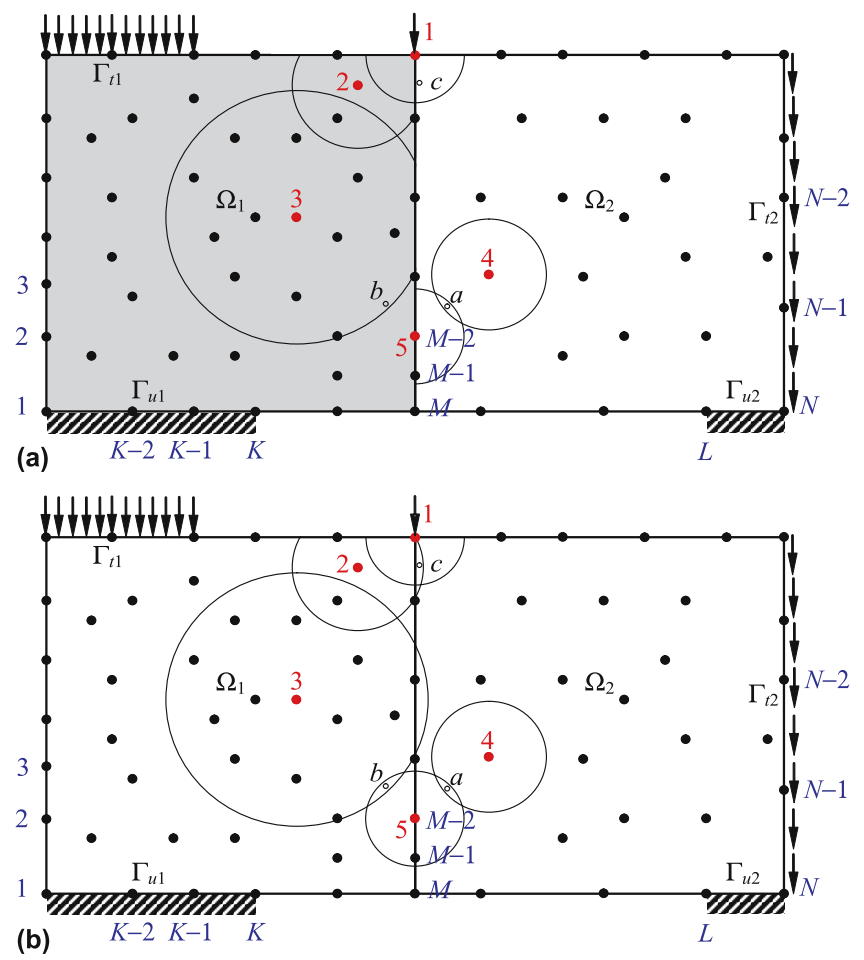

Figure 3. Domains of influence for nodes and nearest neighbours: (a) Inhomogeneous materials; and (b) Homogeneous materials.

Using the algorithm described above it can be seen that it is easy to follow the damage growth, as well as the stresses and strains in the body.

\section{Treatment of material discontinuities}

The method explained above can be easily extended for solving problems with material discontinuities to study the how the presence of initial damage and damage growth upon loading will affect the interface stresses and strains. For an inhomogeneous body consisting of two different materials in domain $\Omega$, bounded by sub-domains $\Omega_{1}$ and $\Omega_{2}$ as shown in figure $3 a$, equation (20) can be rewritten as

$$
\begin{gathered}
\int_{\Omega_{1}}(1-D) \delta \boldsymbol{\varepsilon}^{T} \tilde{\boldsymbol{\sigma}} d \Omega+\int_{\Omega_{2}}(1-D) \delta \boldsymbol{\varepsilon}^{T} \tilde{\boldsymbol{\sigma}} d \Omega-\int_{\Omega_{1}} \delta \boldsymbol{u}^{T} \boldsymbol{b} d \Omega-\int_{\Omega_{2}} \delta \boldsymbol{u}^{T} \boldsymbol{b} d \Omega \\
-\int_{\Gamma_{t_{1}}} \delta \boldsymbol{u}^{T} \overline{\boldsymbol{t}} d \Gamma-\int_{\Gamma_{t_{2}}} \delta \boldsymbol{u}^{T} \overline{\boldsymbol{t}} d \Gamma=0
\end{gathered}
$$

with $\Gamma_{t_{1}}$ and $\Gamma_{t_{2}}$ being the portions of traction boundary $\Gamma_{t}$ belonging to sub-domains $\Omega_{1}$ and $\Omega_{2}$. Similarly, $\Gamma_{u_{1}}$ and $\Gamma_{u_{2}}$ are the portions of displacement boundary $\Gamma_{u}$ belonging to subdomains $\Omega_{1}$ and $\Omega_{2}$. Note that even though for simplicity of explanation an inhomogeneous body is considered to be made up of two sub-domains $\Omega_{1}$ and $\Omega_{2}$, the concepts developed here can be extended even if domain $\Omega$ is made up of more than two sub-domains. 
The stiffness equation system is assembled in each of sub-domains separately, noting that all points contained in material 1 can only be influenced by nodes in material 1 plus interface nodes; and, all points contained in material 2 can only be influenced by nodes contained in material 2 plus interface nodes. Figures $3 \mathrm{a}$ and $3 \mathrm{~b}$, respectively illustrate the difference in selection of the neighbours to a point in domain $\Omega$, when sub-domains $\Omega_{1}$ and $\Omega_{2}$ are considered to be of different materials (inhomogeneous materials) and when sub-domains $\Omega_{1}$ and $\Omega_{2}$ are considered to be of same materials (homogeneous materials). When sub-domains $\Omega_{1}$ and $\Omega_{2}$ are considered to be of different materials, the domains of influence for nodes completely inside sub-domains $\Omega_{1}$ and $\Omega_{2}$ are truncated at the interface, leaving the domains of influence for an interface node belonging to both sub-domains $\Omega_{1}$ and $\Omega_{2}$ unaffected.

From equation (35), the MLS approximation of $u_{i}\left(\boldsymbol{x}_{J}\right)$ is

$u_{i}^{h}\left(\boldsymbol{x}_{J}\right)=\left\{\begin{array}{l}\sum_{I_{1}=1}^{M} \sum_{I_{2}=1}^{M} \Phi_{I_{1}}\left(\boldsymbol{x}_{J}\right) \Lambda_{I_{1} I_{2}}^{\prime(1)^{-1}} d_{I_{2}}^{i}=\boldsymbol{\Phi}_{J}^{i T} \boldsymbol{\Lambda}^{\prime(1)^{-1}} \boldsymbol{d}^{(1)} \quad \text { if } \boldsymbol{x}_{J} \in \Omega_{1} \\ \sum_{I_{1}=M-2}^{N} \sum_{I_{2}=M-2}^{N} \Phi_{I_{1}}\left(\boldsymbol{x}_{J}\right) \Lambda_{I_{1} I_{2}}^{\prime(2)-1} d_{I_{2}}^{i}=\Phi_{J}^{i T} \Lambda^{\prime(2)^{-1}} \boldsymbol{d}^{(2)} \quad \text { if } \boldsymbol{x}_{J} \in \Omega_{2}\end{array}\right.$

where

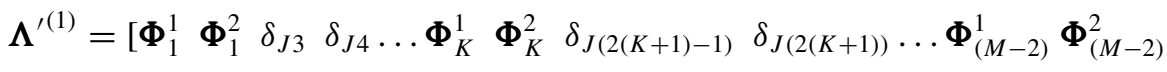

$$
\begin{aligned}
& \left.\boldsymbol{\Phi}_{(M-1)}^{1} \boldsymbol{\Phi}_{(M-1)}^{2} \boldsymbol{\Phi}_{M}^{1} \boldsymbol{\Phi}_{M}^{2}\right]^{T} \in \mathcal{L}\left(\Re^{2 M} \times \Re^{2 M}\right),
\end{aligned}
$$

and

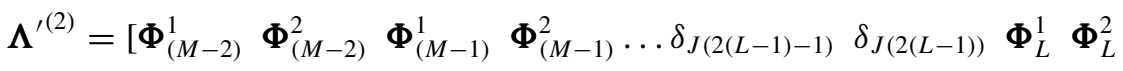

$$
\begin{aligned}
& \left.\delta_{J(2(L+1)-1)} \delta_{J(2(L+1))} \ldots \boldsymbol{\Phi}_{(N-1)}^{1} \boldsymbol{\Phi}_{(N-1)}^{2} \boldsymbol{\Phi}_{N}^{1} \boldsymbol{\Phi}_{N}^{2}\right]^{T} \\
& \in \mathcal{L}\left(\Re^{2(N-M)} \times \Re^{2(N-M)}\right),
\end{aligned}
$$

are the scaled transformation matrices for the domains $\Omega_{1}$ and $\Omega_{2}$ respectively. Using equation (52), by following the similar procedures as explained in section $4 \cdot 1$, stiffness equation system similar to equation (38) can be formulated for each of the sub-domains and can be assembled along the interface nodes to get the global stiffness equation system (Arun et al 2009) and can solve for the increment in nodal parameters and strain increment in each of the sub-domains. The state determination procedure is similar to the one explained in section 4.2 , except that for each of the sub-domains it has to be evaluated separately.

\section{Numerical examples}

To demonstrate the performance of EFGM described above, three numerical examples are presented. In all the numerical examples material is treated as linear elastic, linear kinematic hardening. The linear basis with the student's $t$-distribution weight function (Rao \& Rahman 2000 ) is adopted in EFGM analysis. The scaling parameter, $z_{\max }$ (Rao \& Rahman 2000) and the student's $t$-distribution weight function (Rao \& Rahman 2000) parameter, $\beta$ are taken as 2.01. For numerical integration, one point and $8 \times 8$ Gauss quadrature is adopted respectively for one and two-dimensional numerical examples presented below. Numerical examples involving both with and without material discontinuities are solved. 


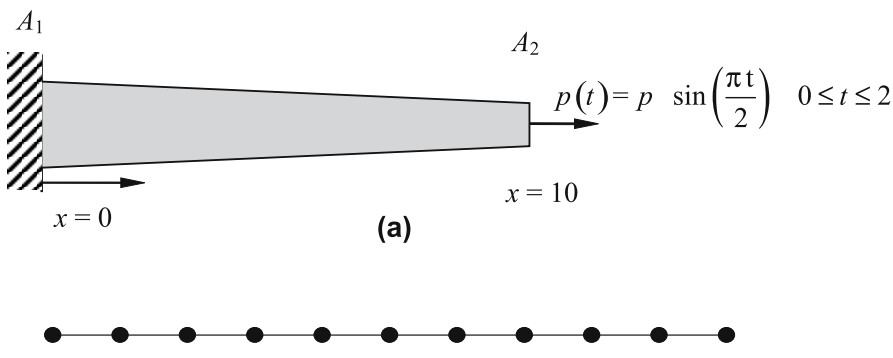

(b)

Figure 4. Bar subjected to sinusoidal load at the free end: (a) Geometry and loads; and (b) Meshless discretization (11 nodes).

\subsection{Example 1. One-dimensional bar}

Consider a bar with length, $L=10 \mathrm{~mm}$ and cross sectional areas, $A_{1}$ and $A_{2}$ respectively at $x=0 \mathrm{~mm}$ and $x=10 \mathrm{~mm}$ as shown in figure $4 \mathrm{a}$. Bar is fixed at $x=0 \mathrm{~mm}$ and is free at $x=10 \mathrm{~mm}$. Sinusoidal load, $p(t)=p \sin (\pi t / 2) 0 \leq t \leq 2$ is applied at the free end of the bar, with $p=320 \mathrm{~N}$. Material properties used in the numerical study are as follows: $E_{0}=200 \mathrm{GPa}, h=10 \mathrm{GPa}, v=0.3, \sigma_{y}=250 \mathrm{MPa}, S=0.06, s=1, D_{c}=0.3$ and damage threshold value, $\varepsilon_{p D}$ is taken as zero. In the numerical analysis both the cases of uniform cross sectional area (with $A_{1}=A_{2}=1 \mathrm{~mm}^{2}$ ), and linearly varying cross sectional area (with $A_{1}=2 \mathrm{~mm}^{2}$ and $A_{2}=1 \mathrm{~mm}^{2}$ ), are studied assuming the following two cases of initial damage: (i) uniform initial damage, $D_{0}=0.12$ and (ii) varying initial damage, $D_{0}(x)=$ $0.12 \sin (\pi x / L)$, which results in the following four cases: (a) uniform cross sectional area (UA) and uniform initial damage (UD); (b) uniform cross sectional area (UA) and varying initial damage (VD); (c) linearly varying cross sectional area (VA) and uniform initial damage (UD); and (d) linearly varying cross sectional area (VA) and varying initial damage (VD). A typical meshless discretization involving 11 uniformly spaced nodes is shown in figure $4 \mathrm{~b}$. In addition, to validate the proposed method finite element analysis has been carried out for all the four cases with same number of nodes (11 nodes) using 10, two-node linear elements.

Figure 5 shows the predicted axial displacement at the free end as a function of number of nodes $N$. The results of all the four cases are shown. Indeed, the proposed meshless method generates convergent solutions of the axial displacement at the free end.

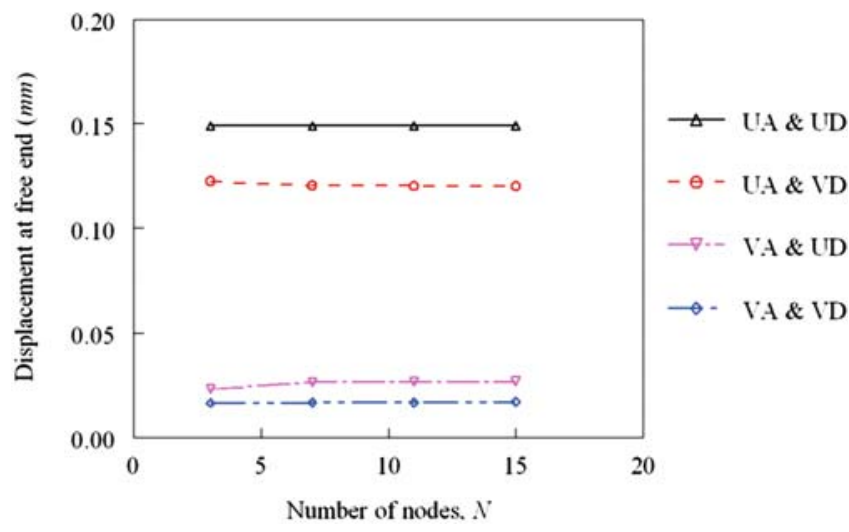

Figure 5. Convergence of axial displacement at the free end of bar. 


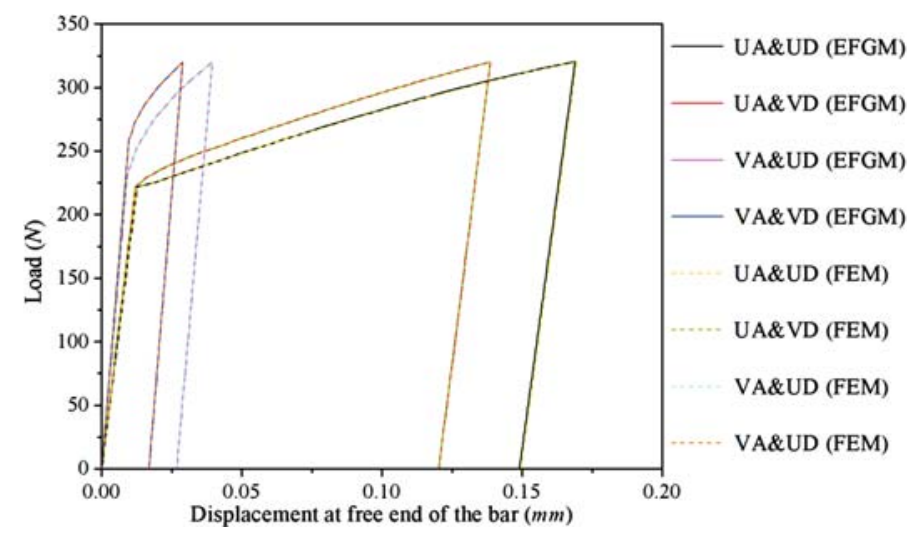

Figure 6. Displacement at the free end of bar during loading and unloading.

Figure 6 shows displacement at the free end of the bar during loading and unloading, for all the four cases. It can be observed from figure 6 that the proposed EFGM is able to produce results which are matching well with the results obtained using FEM. As expected for the case of UA \& UD the bar is less stiff than the other three cases. In the cases of (b), (c) and (d) nonuniform yielding occurs along the length of the bar, which leads to the higher stiffness. It can be observed for figure 6 that the smoothness of displacement variation in shifting from elastic to plastic part increases as the amount of non-uniformity (in geometry and initial damage) increases.

Figure 7 shows stress at $x=10 \mathrm{~mm}$ as a function strain during loading and unloading, for all the four cases. For both the cases of (UA and UD) and (UA and VD), the stress is same at any section for same loading, however the corresponding strain is less in non-uniform initial damage case. Similarly, for both the cases of (VA and UD) and (VA and VD), the stress is same at any section for same loading, however the corresponding strain is less in non-uniform initial damage case. It can be observed from figure 7 that for the same initial damage the slope of stress versus strain for UA and VA is same. The yield stress for the case with UD is less than that with VD. Also, it can be observed that even though elastic and hardening modulus are

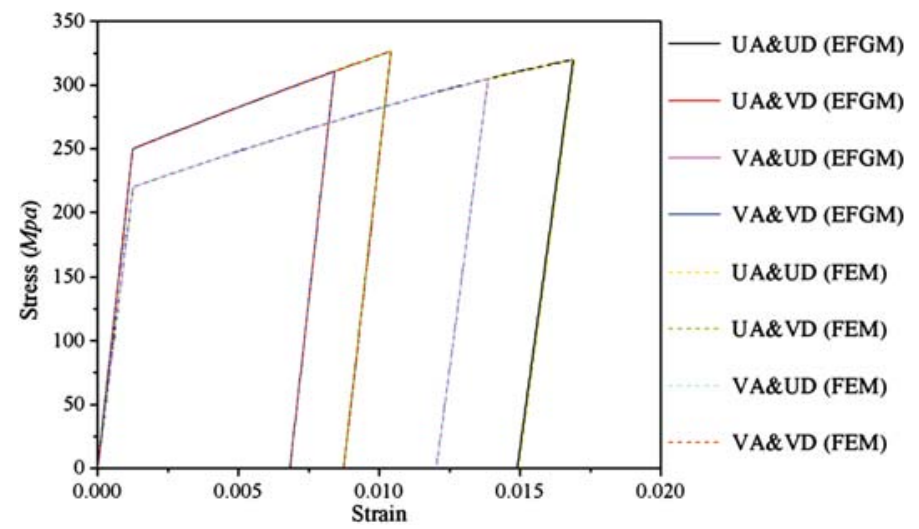

Figure 7. Stress versus strain at $x=10 \mathrm{~mm}$ during loading and unloading. 


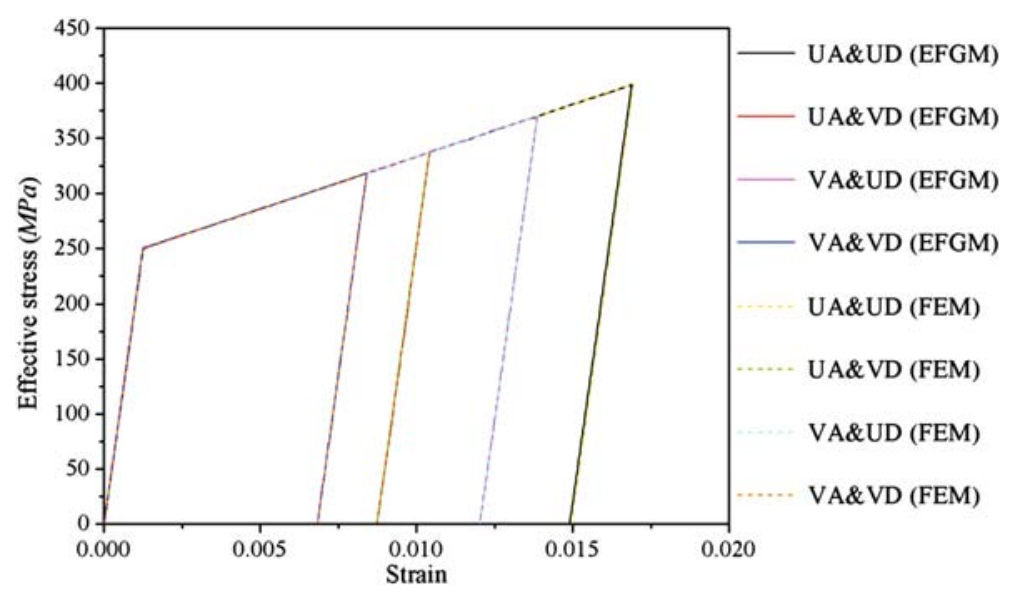

Figure 8. Effective stress versus strain at $x=10 \mathrm{~mm}$ during loading and unloading.

same, the residual strain is different for each of the four cases due to non-uniform yielding, plastic strain and damage growth.

Figure 8 shows effective stress at $x=10 \mathrm{~mm}$ as a function strain during loading and unloading, for all the four cases. Since effective stress is the actual stress in the body, which follows the defined material property the slope of elastic and hardening part is same for all the four cases as that of the virgin material $\left(E_{0}\right.$ and $\left(E_{0} h\right) /\left(E_{0}+h\right)$ respectively) which is independent of geometry and initial damage variation. But the stress developed due to the same applied load varies for each of the four cases, since it depends on the effective resisting area available which depends on geometry of the bar and also on the amount of damage evolved at the point of consideration.

It can be observed from both figures 7 and 8 that the proposed elasto-plastic EFGM for damage analysis is predicting results which are matching well with the corresponding FEM solutions.

Effect of material discontinuity is studied by assuming elastic modulus to be equal to $E_{1}$ and $E_{2}$ respectively for $0 \leq x \leq 10 \mathrm{~mm}$ and $5 \leq x \leq 10 \mathrm{~mm}$. This results in the bimaterial bar with material discontinuity at $x=5 \mathrm{~mm}$. All other material properties are assumed to be same for both $0 \leq x \leq 5 \mathrm{~mm}$ and $5 \leq x \leq 10 \mathrm{~mm}$, and are taken as, $h_{1}=h_{2}=10 \mathrm{GPa}$, $v=0 \cdot 3, \sigma_{y}=250 \mathrm{MPa}, S=0 \cdot 06, s=1, D_{c}=0.3$ and damage threshold value, $\varepsilon_{p D}$ is taken as zero. Both the materials are assumed to have same initial damage, $D_{0}=0 \cdot 12$.

Figure 9 shows the axial displacement as a function of $x$ at the end of unloading, obtained by the proposed elasto-plastic EFGM, with damage (WD) and without damage (ND) for different values of $E_{1}$ and $E_{2}$. It can be observed from figure 9 that if the damage is neglected in the analysis, the axial displacement along the length is independent of elastic modulus and material discontinuities since kinematic hardening modulus is assumed to be same through out the length. But when damage is included in the analysis, the axial displacement along the length shows dependency on the elastic modulus even if kinematic hardening modulus is assumed to be same through out the length. Also presence of discontinuity introduces change in the slope at $x=5 \mathrm{~mm}$ in a damage coupled analysis as shown in figure 9 .

Figure 10 shows displacement at the free end of the bar during loading and unloading, obtained by the proposed elasto-plastic EFGM, with damage (WD) and without damage (ND) 


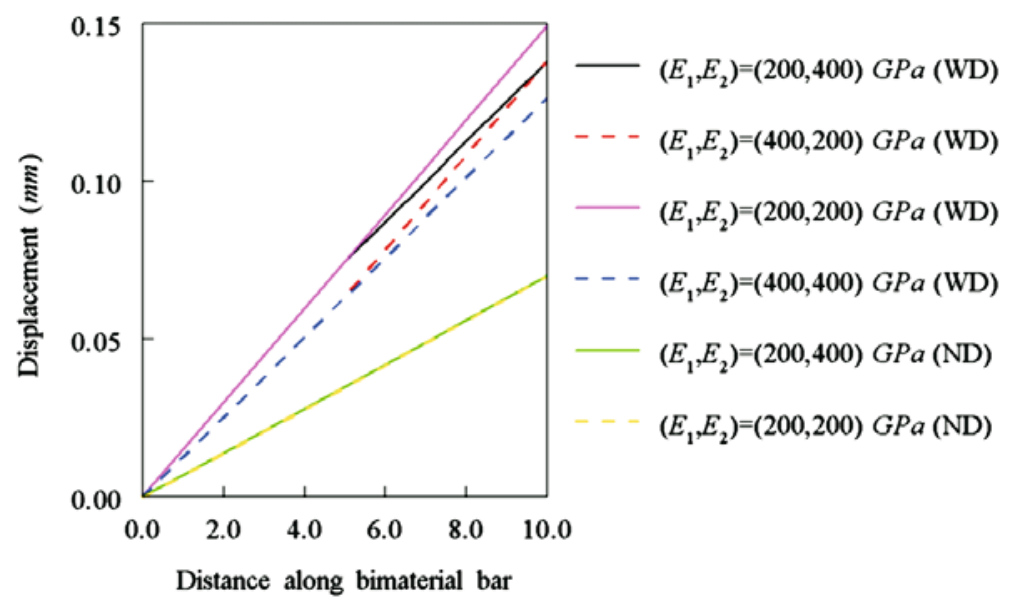

Figure 9. Displacement along the length of bimaterial bar during loading and unloading.

for different values of $E_{1}$ and $E_{2}$. It can be observed from figure 10 that the bimaterial bar with different $E_{1}$ and $E_{2}$ shows mean response of the bimaterial bar with same $E_{1}$ and $E_{2}$. Figure 10 also shows that the analysis performed without considering the effect of damage leads to an over estimation of stiffness of the bimaterial bar.

\subsection{Example 2. Two-dimensional plane stress problem}

Two-dimensional plane stress problem with monotonic loading is studied by taking a bimaterial plate with dimension $L=10 \mathrm{~mm}$ and loading with $p=320 \mathrm{~N}$, as shown in figure 11a. Uniform initial damage $D_{0}=0 \cdot 12$ is assumed. A typical meshless discretization involving a total of 49 uniformly spaced nodes is shown in figure $11 \mathrm{~b}$.

Figure 12 shows the predicted displacement along the edge $x_{2}=10 \mathrm{~mm}$ as a function of number of meshless nodes, $N(=25,49,81$ and 121). Indeed, the proposed meshless method generates convergent solutions of the displacement.

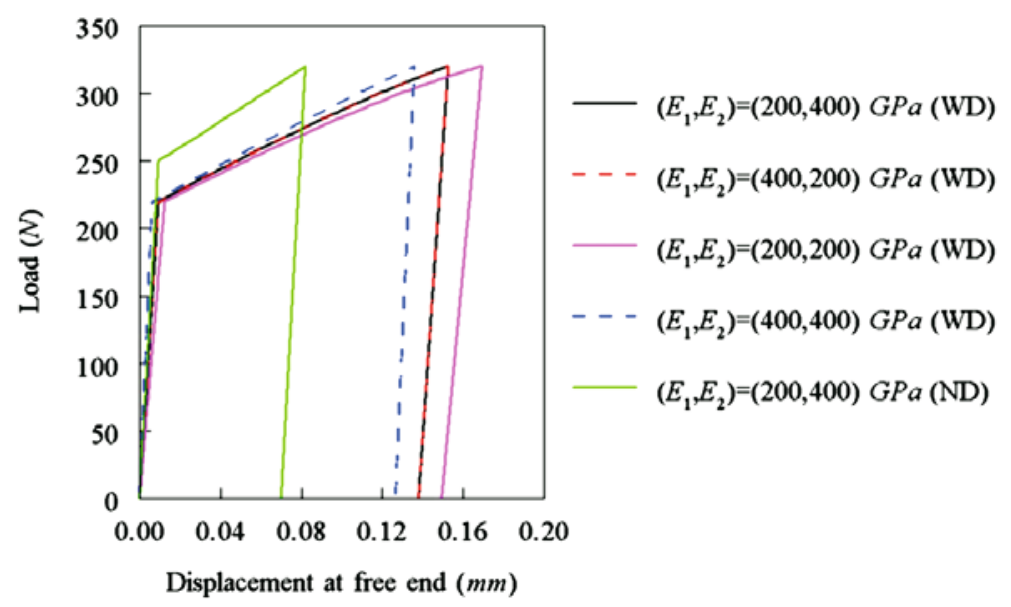

Figure 10. Displacement at the free end of bimaterial bar during loading and unloading. 

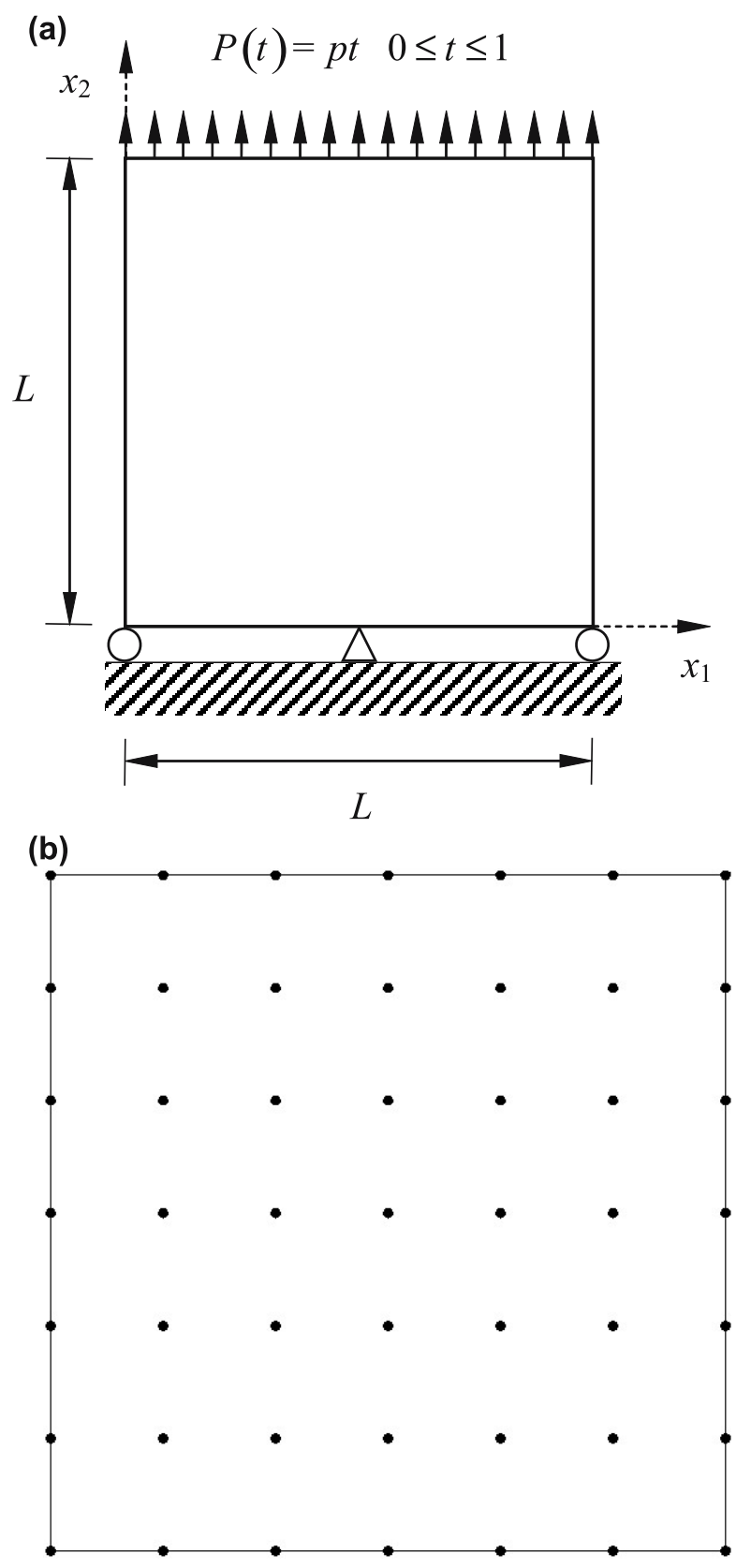

Figure 11. Square plate subjected to uniformly distributed tension: (a) Geometry and loads; and (b) Meshless discretization (49 nodes).

Effect of material discontinuity is studied by assuming elastic modulus to be equal to $E_{1}$ and $E_{2}$ respectively for $0 \leq x_{2} \leq 5 \mathrm{~mm}$ and $5 \leq x_{2} \leq 10 \mathrm{~mm}$. This results in the bimaterial plate with material discontinuity at $x_{2}=5 \mathrm{~mm}$. All other material properties are assumed to be same for both $0 \leq x_{2} \leq 5 \mathrm{~mm}$ and $5 \leq x_{2} \leq 10 \mathrm{~mm}$, and are taken as, $h_{1}=h_{2}=10 \mathrm{GPa}, v=0 \cdot 3, \sigma_{y}=250 \mathrm{MPa}, S=0 \cdot 06, s=1, D_{c}=0 \cdot 3$. 

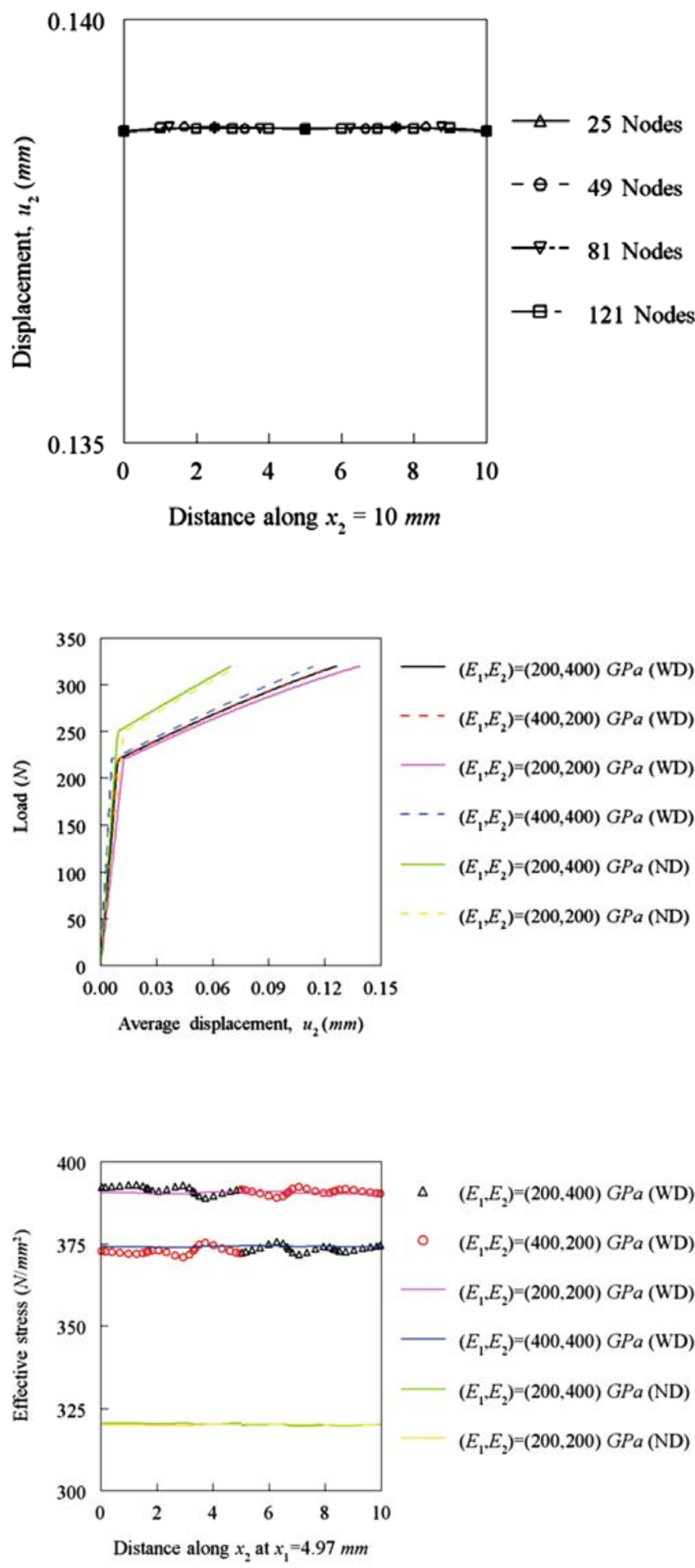

Figure 12. Convergence of displacement along the edge $x_{2}=$ $10 \mathrm{~mm}$.

Figure 13. Average displacement along the edge $x_{2}=10 \mathrm{~mm}$ at the end of monotonic loading.

Figure 14. Effective stress along the line $x_{1}=4.97 \mathrm{~mm}$ at the end of monotonic loading. 

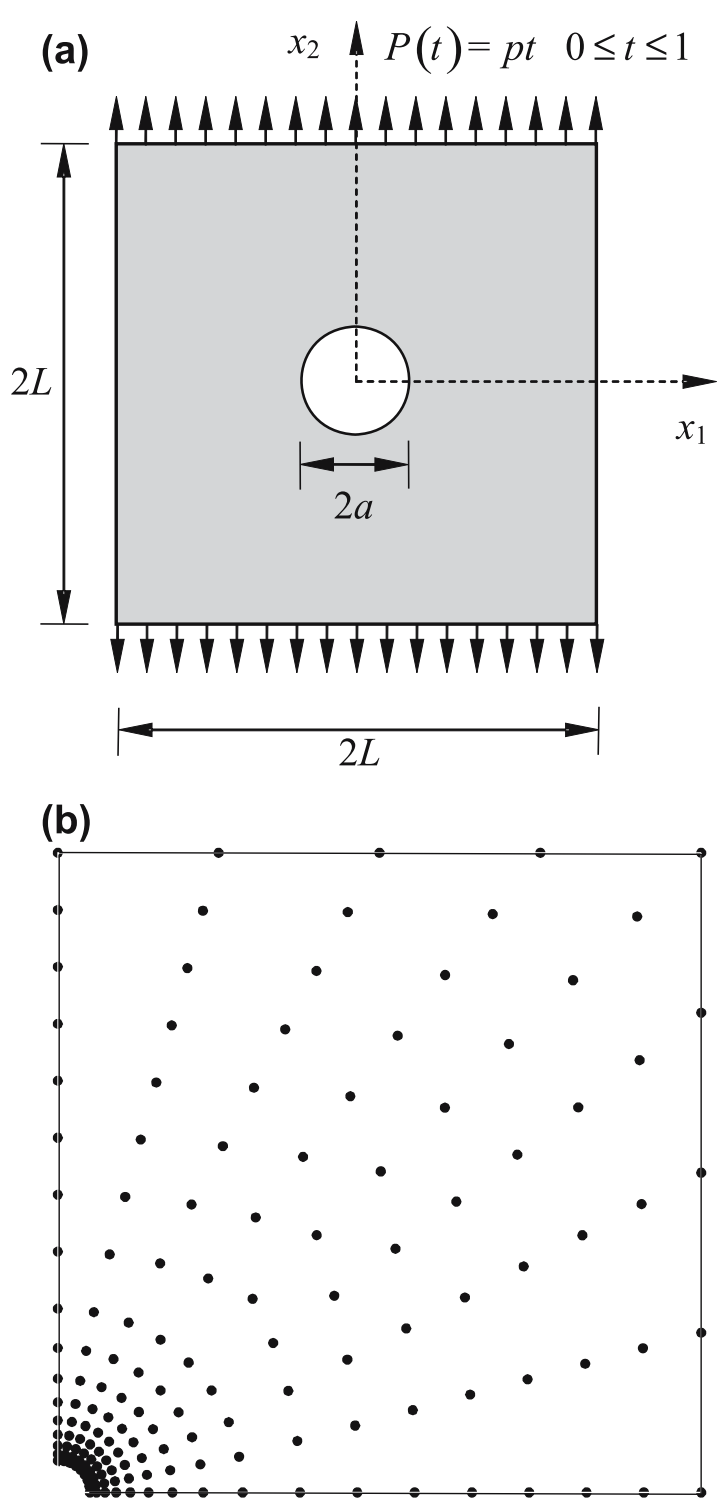

Figure 15. Plate with hole under tension: (a) Geometry and loads; and (b) Meshless discretization (153 nodes).

Figure 13 shows average displacement along the edge $x_{2}=10 \mathrm{~mm}$ at the end of the monotonic loading, obtained by the proposed elasto-plastic EFGM, with damage (WD) and without damage (ND) for different values of $E_{1}$ and $E_{2}$. It can be observed from figure 13 that the bimaterial plate with different $E_{1}$ and $E_{2}$ shows mean response of the bimaterial plate with same $E_{1}$ and $E_{2}$. Figure 13 also shows that the analysis performed without considering the effect of damage leads to an over estimation of stiffness of the bimaterial plate.

Figure 14 shows effective stress as a function of $x_{2}$ along the line $x_{1}=4.97 \mathrm{~mm}$ at the end of the monotonic loading, obtained by the proposed elasto-plastic EFGM, with damage 
(WD) and without damage (ND) for different values of $E_{1}$ and $E_{2}$. It can be observed from figure 14 that material discontinuity results in effective stress jump at the interface. Different values of $E_{1}$ and $E_{2}$ resulted oscillations in predicted effective stress. These oscillations in predicted effective stress are observed to be reduced with increased refinement of meshless discretization. From figure 14 it can be observed that in the analysis without considering damage effective stress is same as stress and that the stress equilibrium across the interface is satisfied as expected.

\subsection{Example 3. Plate with a hole under tension}

Square plate with circular hole under far field tensile loading as shown in figure $15 \mathrm{a}$ is considered. Plate with dimensions $2 L=40 \mathrm{~mm}$ and $2 a=2 \mathrm{~mm}$ is considered. A monotonically increasing uniformly distributed load with $p=225 \mathrm{~N}$ is applied as shown in figure 15a. Due to symmetry only one quarter of the plate is analysed. As this problem is sensitive to nodal distribution due to the presence of geometric discontinuity at the hole, pure elastic analysis is carried out adopting different meshless nodal discretizations, before performing elasto-plastic EFGM damage evolution analysis. A uniformly distributed load of magnitude, $p=1 \mathrm{~N}$ and an initial damage value $D_{0}=0$ is used for elastic analysis. Figure 16 compares the normal stress, $\sigma_{22}$ along $x_{2}=0$ obtained using different meshless nodal discretizations with the theory of elasticity solution (Timoshenko \& Goodier 1970). A typical meshless discretization with 153 nodes is shown in figure 15b. Discontinuity in the geometry results non-uniform stress and plastic strain evolution in the plate which in tum leads to localized damage evolution near the hole. Figure 17 shows the variation of average displacement with load, along the free end of the plate, $x_{2}=20 \mathrm{~mm}$ for different initial damage values. It can be observed from figure 17 that there is a smooth variation from elastic to plastic part in stress-strain curves due to non-uniform plastic evolution. Figure 17 also shows the variation of average displacement with load obtained from EFGM analysis by neglecting damage effect. It is clear from figure 17 that the presence of damage will produce a considerable change in the material

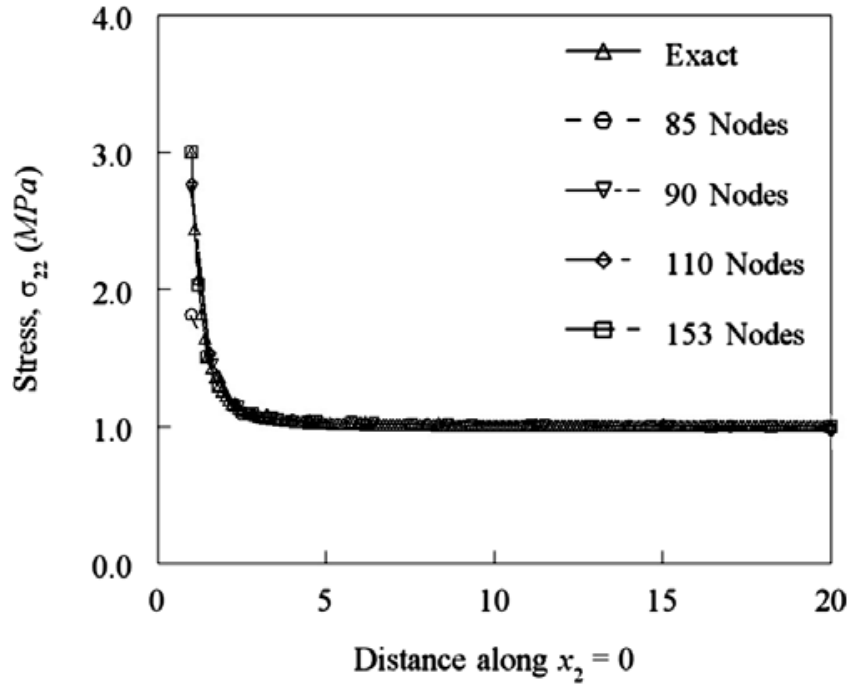

Figure 16. Normal stress $\sigma_{22}$ along $x_{2}=0$ for plate with hole under tension. 


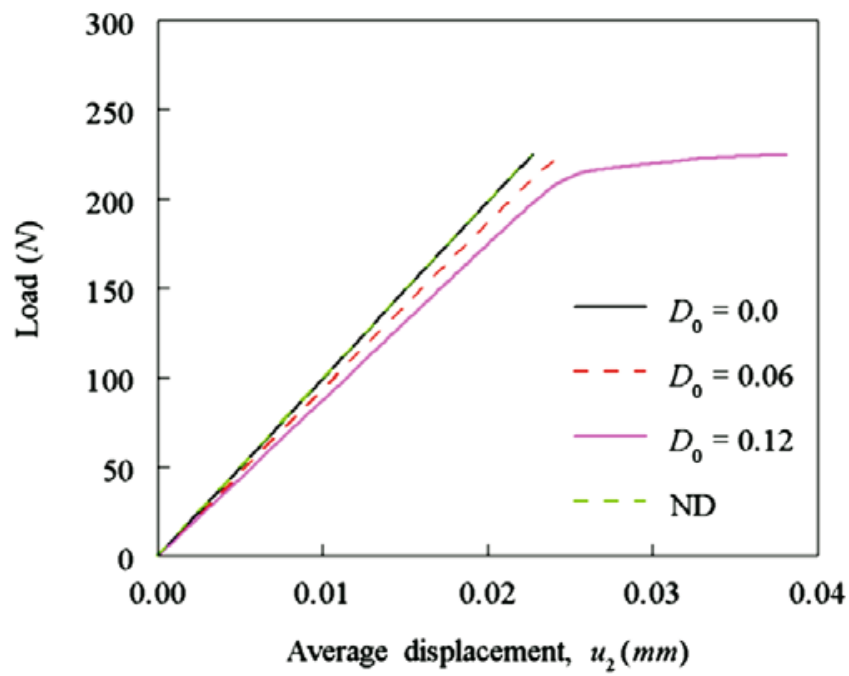

Figure 17. Variation of average displacement with load along the free end of plate, $x_{2}=20 \mathrm{~mm}$.

behaviour. Figure 18 shows damage level over the entire domain at the end of the loading. It can be observed from figure 18 that the proposed numerical method is able to capture the damage concentration near the hole.

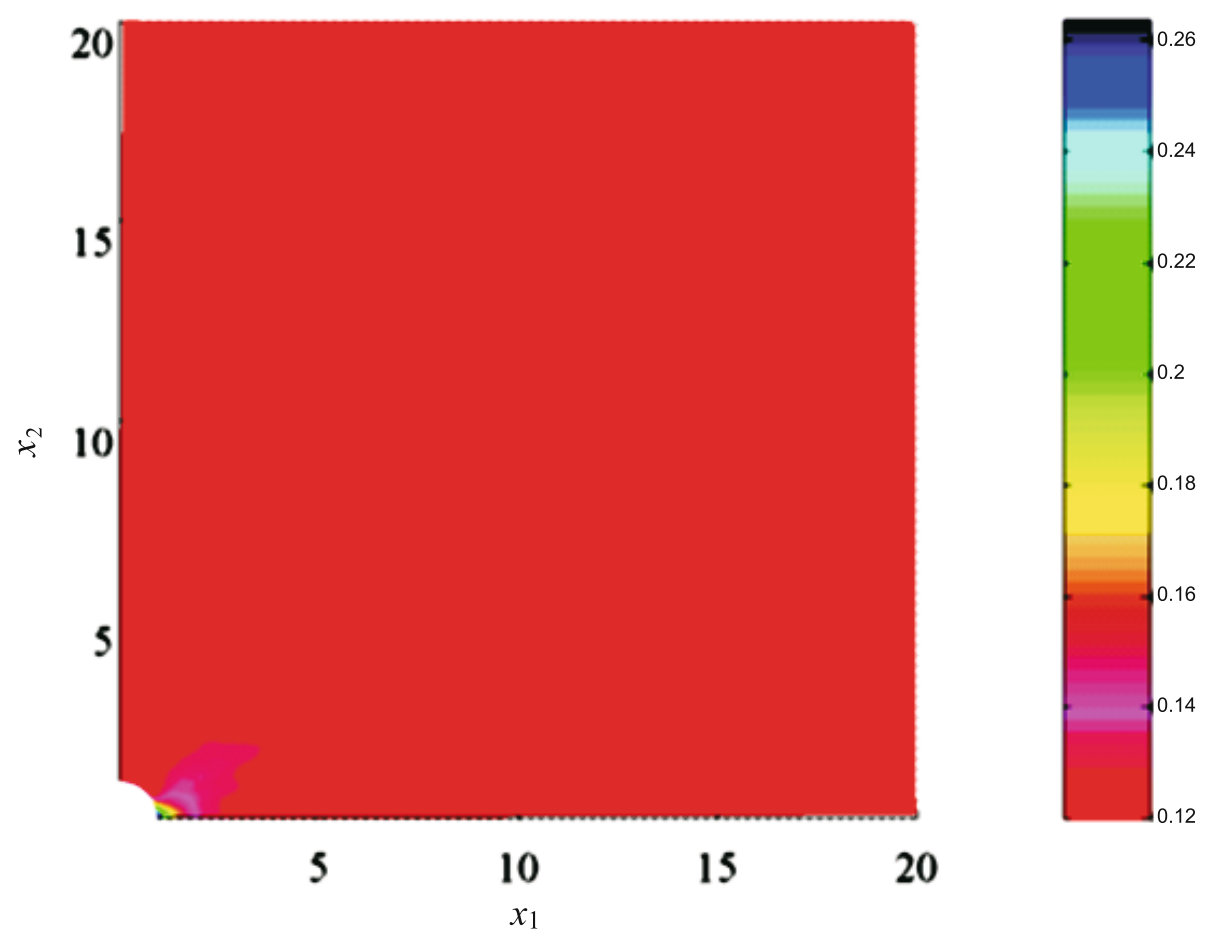

Figure 18. Damage distribution over entire domain of plate with hole at the end of loading. 


\section{Conclusions}

A study has been carried out using the proposed EFGM to understand the effect of initial damage and its growth on structural response of single and bi-material problems. A simple method is adopted for enforcing the EBCs in EFGM. Isotropic unified damage law is used to model the damage growth. Three numerical examples comprising of one-and two-dimensional problems are solved to illustrate the effectiveness of the proposed method in analysis of uniform and non-uniform damage evolution problems. Both the cases of uniform cross sectional area, and linearly varying cross sectional area assuming uniform and varying initial damage, are considered in the numerical study. Numerical results shown are given below.

- The study substantiate the fact that it is necessary to consider initial damage and damage growth in the analysis since the presence of damage reduces the stiffness of the material leading to unexpected material response and premature yielding.

- Material discontinuity results in effective stress jump at the interface. Also when damage is included in the analysis, the axial displacement along the length shows dependency on the elastic modulus even if kinematic hardening modulus is assumed to be same through out the length. Neglecting these effects certainly can lead to an unsafe design which is by no means is acceptable for any engineering structures.

- The proposed method is able to capture the damage localizations and its effect on structural responses, due to non-uniformity in geometry or initial damage variation or both.

- In addition the proposed EFGM is able to capture damage concentration in problems with stress concentrations.

\section{References}

Alves M K, Rossi R 2003 A modified element-free Galerkin method with essential boundary conditions enforced by an extended partition of unity finite element weight function. Int. J. Numerical Methods in Eng. 57: 1523-1552

Arun C O, Rao B N, Sivakumar S M 2007 A new material discontinuity treatment in element free Galerkin method. In: Proceedings of ICTACEM, 27-29 December, India

Arun C O, Rao B, Sivakumar S M 2009 On material discontinuity modeling in element free Galerkin method. J. Struct. Eng., SERC 36(3): 182-194

Belytschko T, Lu Y Y, Gu L 1994 Element-free Galerkin methods. Int. J. Numerical Methods in Eng. 37: 229-256

Belytschko T, Lu Y Y, Gu L 1995 Crack propagation by element-free Galerkin methods. Eng. Fracture Mech. 51(2): 295-315

Benallal A Rene B, Doghri I 1988 An integration algorithm and the corresponding consistent tangent operator for fully coupled elasto plastic and damage equations. Communications in Applied Numerical Methods 4: 731-740

Bhatti A M 2006 Advanced topics in finite element analsyis of structures (Hoboken, NJ: John Wiley and Sons)

Chaboche J L 1988 Continuum damage mechanics: Part I - General concepts. J. Applied Mech. 55: 59-64

Chaboche J L 1988 Continuum damage mechanics: Part II - Damage growth, Crack initiation, and Crack growth. J. Applied Mech. 55: 59-64

Chen J S, Wang H P 2000 New Boundary Condition Treatments in Mesh-free Computation of Contact Problems. Computer Methods in Applied Mech. and Eng. 187: 441-468

Chen W F, Han D J 1988 Plasticity for Structural Engineers (New York, USA: Springer-Verlag) 
Dolbow J, Belytschko T 1998 An introduction to programming the meshless element free Galerkin method. Archives of Computational Mechanics 15(3): 207-241

Duarte C A M, Oden J T $1996 H-p$ Clouds - An $h-p$ meshless method. Numerical Methods for Partial Differential Equations 12(6): 673-705

Kachanov L M 1958 Time of the rupture process under creep conditions. IVZ Akad Nauk, S. S. R. Otd Tecg Bayj, 8: 26-31

Kattan P I, Voyiadjis G Z 2002 Damage mechanics with finite elements practical applications with computer tools (Berlin, Heidelberg, Germany: Springer-Verlag)

Krongauz Y, Belytschko T 1996 Enforcement of essential boundary conditions in meshless approximation using finite elements. Computer Methods in Applied Mechanics and Eng. 131: 1335-1345

Lancaster P, Salkauskas K 1981 Surfaces generated by moving least squares methods. Mathematics of Computation 37: 141-158

Lemaitre J 1971 Evaluation and dissipation of damage in metals submitted to dynamic loading. In: Proceedings I.C.M.I. Kyoto, Japan

Lemaitre J 1985 A continuous damage mechanics model for ductile fracture. J. Eng. Materials and Technol. 107: 83-89

Lemaitre J 1992 A course on damage mechanics (Berlin, Heidelberg, Germany: Springer-Verlag)

Lemaitre J, Desmorat R 2005 Engineering damage mechanics (Berlin, Heidelberg, Germany: Springer-Verlag)

Liu W K, Jun S, Zhang Y F 1995 Reproducing kernel particle methods. Int. J. Numerical Methods in Fluids 20: 1081-1106

Liu W K, Li S, Belytschko T 1997 Moving least square kernel Galerkin method - Part I: Methodology and convergence. Computer Methods in Applied Mechanics and Eng. 143: 422-433

Liu G R 2002 Mesh free methods: Moving beyond the finite element method (LLC, Boca Raton FL, USA: CRC Press)

Li G, Belytschko T 2001 Element-free Galerkin methods for contact problems in metal forming analysis. Eng. Computations 18: 62-78

Lucy L 1977 A numerical approach to testing the fission hypothesis. Astron. J. 82: 1013-1024

Lu Y Y, Belytschko T, Gu L 1994 A new implementation of the element free Galerkin method. Computer Methods in Applied Mechanics and Eng. 113: 397-414

Lu Y Y, Belytschko T, Tabbara M 1995 Element-free Galerkin methods for wave propagation and dynamic fracture. Computer Methods in Applied Mechanics and Eng. 126: 131-153

Melenk J M, Babuska I 1996 The partition of unity finite element method: Basic theory and applications. Computer Methods in Applied Mechanics and Eng. 139: 280-314

Monaghan J J 1988 An introduction to SPH. Computer Physics Communications 48: 89-96

Nagashima T 1999 Node-by-Node meshless approach and its applications to structural analyses. Int. J. Numerical Methods in Eng. 46: 341-385

Nayroles B, Touzot G, Villon P 1992 Generalizing the finite element method: Diffuse approximation and diffuse elements. Computational Mechanics 10: 307-318

Ponthot J P, Belytschko T 1998 Arbitrary lagrangian-eulerian formulation for element-free Galerkin method. Computer Methods in Applied Mechanics and Eng. 152: 19-46

Rabotnov Y N 1969 Creep Problems of Structural Members. (North Holland, Amsterdam)

Rao B N, Rahman S 2000 An efficient meshless method for fracture analysis of cracks. Computational Mechanics 26(4): 398-408

Sivakumar M S, Voyiadjis G Z 1997 A simple implicit scheme for stress response computation in plasticity models. Computational Mechanics 20: 520-529

Timoshenko S P, Goodier J N 1970 Theory of Elasticity. (New York, USA: McGraw-Hill)

Voyiadjis G Z, Kattan P I 1990 A coupled theory of dmage mechanics and finite strain elasto-plasticityII. Damage and Finite Strain Plasticity. Int. J. Eng. Sci. 28(6): 505-524

Voyiadjis G Z, Kattan P I 2005 Damage mechanics (LLC, Boca Raton FL, USA: CRC Press) 